\title{
Modeling search and rescue, medical disaster team response and transporta- tion of patients in Ishinomaki city after tsunami disaster
}

\author{
Erick Mas ${ }^{1, *}$, Shinichi Egawa M.D. ${ }^{1,}$, Hiroyuki Sasaki M.D. ${ }^{1,}$, and Shunichi Koshimura ${ }^{1,}$ \\ ${ }^{1}$ International Research Institute of Disaster Science, Tohoku University, Sendai, Japan
}

\begin{abstract}
The 2011 Great East Japan Earthquake caused massive devastation and numerous affected people. In particular, the Ishinomaki area of the Miyagi Prefecture was one of the hardest hit areas during the event. In addition, due to tsunami inundation, several medical facilities were destroyed or inactive at the time. The Ishinomaki Red Cross Hospital served as the last bastion to respond and attend injuries and illnesses in the disaster. We aim to build an agent-based model of triage and transportation of patients to the hospital. The individual and joint activities of response teams were considered to build the agent architecture and they were incorporated into the model. We model the first three days of response activities within the central Ishinomaki area and evaluated the number of patients transported to the hospital including their evaluated health status. We discuss here the case closest to the 2011 event and evaluate the strategies for search and rescue and patient transportation to speed up medical response in the aftermath of a disaster.
\end{abstract}

\section{Introduction}

In the aftermath of the 2011 Great East Japan Earthquake and Tsunami (GEJET), search and rescue (SAR) operations were massive and complex. SAR activities started locally from firefighters and the police. Similarly, sea search and rescue actions were leaded by the Japan Coast Guard (JCG). In special cases, such as this one, the Japan Self Defense Forces (JSDF) are also involved in the operations. The Japan's Fire and Disaster Management Agency (FDMA) has conducted reviews of rescue activities in response to the GEJET [6] where they summarized the lessons for SAR focusing on the activities at tsunami-hit areas during the "golden 72 hours". The activities conducted by first responders in the fire services include shutting down water gates, delivering tsunami warning to local residents and guiding evacuation. Unfortunately, member volunteers of fire corps died closing tsunami gates in the area [2]. On the following hours of the tsunami impact, local fire departments were aided by less affected inland departments in operations of SAR, fire suppression and injury transportation. SAR activities conducted by fire service personnel, police, JCG and JSDF saved approximately 27,000 people. Koresawa (2013)[6] details the number of rescued people by local fire departments in tsunami-affected areas of Aomori, Iwate, Miyagi, Fukushima and Ibaraki prefectures to be over 3,500 people during the first 48 hours, and later consistently around 200 people every day from the third day towards the end of the month.

The objective of this study is to develop an agent-based model of disaster response incorporating search and rescue, medical assistance evaluation through the Disaster

*Corresponding author e-mail: mas@irides.tohoku.ac.jp
Medical Assistance Team (DMAT) and transportation of patients within the first three days after the event. A case study on the central Ishinomaki area in Japan is simulated following the data from the 2011 Great East Japan Earthquake and Tsunami.

\section{Study Area}

Ishinomaki City (Figure 1) in the Miyagi Prefecture had the largest number of death and missing bodies in the 2011 event $(3,972)$. Also, medical centers were inundated and out of service due to the damage caused to them. Within the Ishinomaki area, one of the main hospitals out of the inundation zone was the Ishinomaki Red Cross Hospital (IRCH) which remained active. At the IRCH the total number of patients in the first month was at least five times the regular number of patients before the earthquake [1]. The IRCH coordinated all medical teams from the Japanese Red Cross Society and other agencies at evacuation centers throughout Ishinomaki city [4]. Several predisaster preparedness activities conducted by the IRCH were key to a better response during the 2011 GEJET [3] On a 2015 report by the IRCH, they reported the situation of first days of transported patients and their condition classified in minor, medium, severe and serious injuries as shown in Table 1

The target area for analysis is shown in Figure 1 within the inundation area. It is bounded by the Old-Kitakami river in the east and north near the Fire Department Headquarters, the channel connecting Old-Kitakami and Jo rivers in the west side and the inundated areas below the $2 \mathrm{~m}$ inundation depth. We focused the study below the 2 $m$ inundation depth since the areas over that level of inun- 
Table 1: Number of patients transported to the Ishinomaki Red Cross Hospital within the first three days after the event classified by injury level. In () are the number corresponding to the target area for this study.

\begin{tabular}{|l|c|c|c|c|c|}
\hline Day & Minor & Medium & Severe & Serious & Total \\
\hline $3 / 12$ & $47(24)$ & $33(16)$ & $17(8)$ & $2(1)$ & $99(49)$ \\
$3 / 13$ & $552(271)$ & $142(70)$ & $74(36)$ & $11(5)$ & $779(382)$ \\
$3 / 14$ & $1,037(509)$ & $152(74)$ & $39(19)$ & $23(11)$ & $1,251(613)$ \\
\hline Total & $1,636(804)$ & $327(160)$ & $130(63)$ & $36(17)$ & $2,129(1,044)$ \\
\hline
\end{tabular}

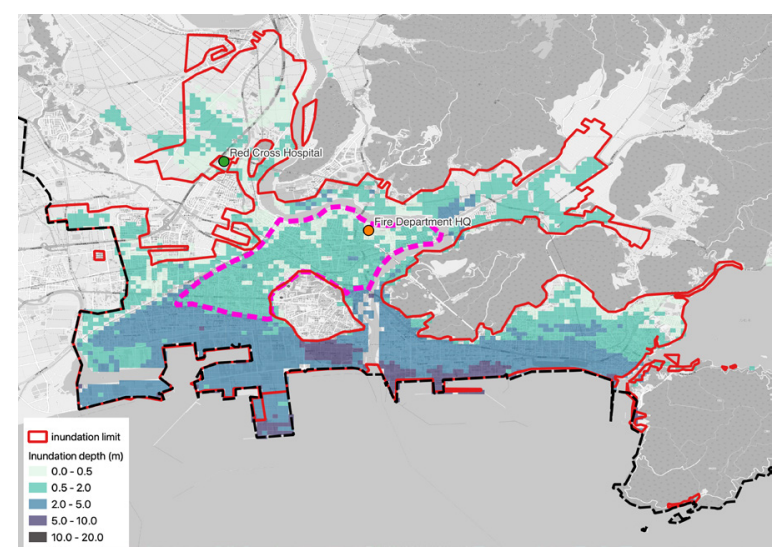

Figure 1: The area of study at Ishinomaki. The inundation limit was provided by the Geospatial Information Authority of Japan. Inundation depths were provided by the Ministry of Land, Infrastructure, Transport and Tourism of Japan. The background is an Open Street Map provided within the QGIS software. The Ishinomaki Red Cross Hospital and the Ishinomaki District Wide-Area Administrative Affairs Union Fire Department HQ are the two points shown in the map. The polygon inset at the center corresponds to the target area for analysis in this study.

dation were heavily damaged and immediate access was reported to be limited due to water presence [6].

Serikawa et al. (2020)[5] showed that the Miyagi Prefectural Police Headquarters reported 3,058 corpses within the whole Ishinomaki area (a larger area that includes Oshika Peninsula and the areas near the Kitakami river). On the other hand, the activity records from the Ishinomaki Wide-Area Administrative Office of the Fire Department reported 2,954 corpses. In addition, within the inundation area shown in Figure 1, 1,448 bodies were found. The rate of bodies within the target area versus the whole Ishinomaki medical area $(1,448 / 2,954=0.49)$ is used as the rate to calculate the number of patients to be transported to the Ishinomaki Red Cross Hospital from the area in study. The values to be used for verification of our model results are shown in parenthesis in Table 1

\section{Materials and Methods}

We compiled several sources detailing the activities following the 2011 GEJET in the Ishinomaki area. In particular, sources related to the IRCH and the triage and transportation of patients [4] (see Table 1). In addition, for the agent architecture and interactions among them we also followed the descriptions and architecture described in [710].

With these, we developed an agent-based model with the aid of the AnyLogic ${ }^{\mathrm{TM}}$ software. AnyLogic is a multimethod simulation tool based on Java language. Some of the simulation paradigms possible to combine within the software are: discrete event or process-centric simulations, system dynamics and agent-based modeling. In this study we focus on the use of agent-based approach. AnyLogic 8.7.5 University edition was used to create and simulate our models.

The model contains four types of agents whose behaviors are summarized in Table 2 and described hereafter.

The first type of agent is called "patient" agent. Within this type a major attribute corresponding to the level of injury makes a distinction on the agent behavior. For instance, a "serious" injury is represented as a black agent and corresponds to a direct casualty from the event. Upon finding this type of agent, SAR team agents will not request for transportation to hospital and leave that agent. In our model assumption, police is in charge of corpses and they will be requested to take care of such agents. Since including such type of agent and activity is out of the scope of our study we did not include a police agent type and the actions taken after finding a serious injury patient is none.

Next, "severe" injury patients are identified as red colored agents in the model. These agents cannot walk but their injuries are threatening to life. Thus, immediate assistance and transportation to medical facilities is needed. When SAR teams find this type of agent, they will request to the Ishinomaki Firefighters Department (IFD) for transportation assistance. A transport team is dispatch from IFD or from nearby locations to carry the patient to the IRCH. Similarly, a "medium" injury patient, a yellow colored agent in the model, has less urgency but similar needs. Then, these type of agent is also carried to the hospital.

Then, within the "patient" agent, those with "minor" injury are represented with a green color in the model and since there is no life-threatening injury, those agents are assumed to be able to walk by themselves and find assistance elsewhere. Within the model once found by the SAR team the agent walks towards the hospital looking for assistance.

Finally, a "DMAT unit" agent is initially located at the IRCH and is in charge of a secondary triage. In this case a reassessment of the urgency of attention is conducted. Thus, a modelled set "severe" agent can be wrongfully triagged as "medium" or viceversa by SAR teams in the field, however, once at the hospital the correct triage level is revealed. Such initial triage inaccuracy can be adjusted in the model in order to reflect and evaluate the effect of low-capacity and training of in-field first responders.

A snapshot of the ABM model is shown in Fig 2. On the right, the Ishinomaki Fire Department marks the start point of SAR teams. The colored dots represent the population and their level of injury or illness based on random selection and statistical information from triage records at the IRCH. SAR activities yield to a first triage when a pa- 
Table 2: Agents' characteristics and initial location for simulation

\begin{tabular}{|c|c|c|c|c|}
\hline & & gent & Initial Location & Description (i.e. triage, task, etc.) \\
\hline \multirow{4}{*}{1} & \multirow{4}{*}{ Patients } & Serious & Random & [Black] Casualty \\
\hline & & Severe & Random & [Red] Cannot walk. Immediate assistance required. \\
\hline & & Medium & Random & [Yellow] Cannot walk. Not urgent but requires assistance. \\
\hline & & Minor & Random & [Green] Can walk. Not a life-threatening injury. \\
\hline 2 & \multicolumn{2}{|c|}{ Firefighter (SAR team) } & IFD & Search and Rescue of patients. Request for transportation. \\
\hline 3 & \multicolumn{2}{|c|}{ Firefighter (Transport team) } & IFD & Pick up and transport to hospital. \\
\hline 4 & \multicolumn{2}{|c|}{ DMAT unit } & IRCH & Triage at hospital. Keeps track of patients. \\
\hline
\end{tabular}

tient is found and then send to the hospital to a second triage by DMAT personnel. Based on the level of injury or illness, the transportation to IRCH is prioritized.

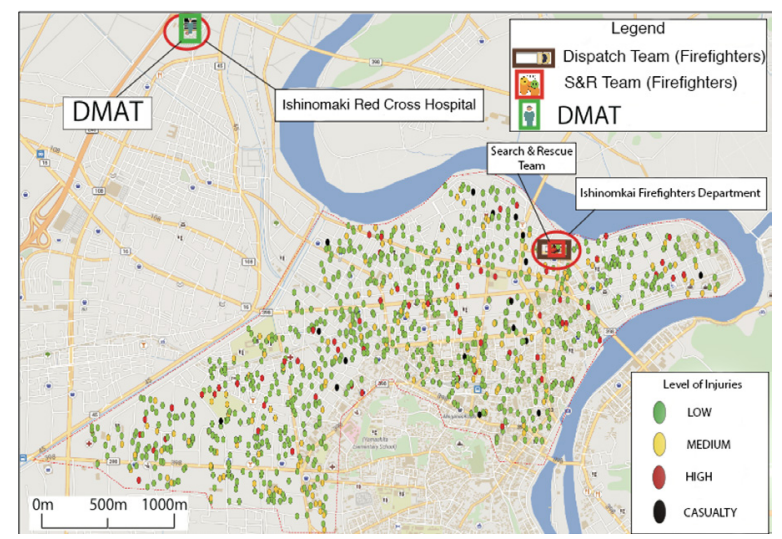

Figure 2: Screen-shot of the developed agent-based model

Regarding the rules for interaction among agents, first, patients are found by SAR teams who conduct a first triage and only severe and medium injury patients are candidates for transportation to the hospital by the Transport team. Requests are stacked and from the resource pool of available vehicles, a Transport team moves to pick up the patient and move together to the IRCH where a DMAT unit is waiting to conduct a second triage. The case of serious injured people is supposed to be taken by another agent (i.e. the police) and their dynamics is out of the scope of this research. Thus, no action is taken in the case of serious injured agents. On the other hand, minor injury patients can move and they are instructed to go to the hospital by their own means.

A particular feature to explore here is the strategy for SAR. This is, the technique employed to explore the area and find survivors. In cases of missing persons hasty searches are common, however in large disaster stricken areas grid search techniques or organized search is necessary. A particular way to organized the SAR operation is through a "roller search", which divides the affected area in subareas for search. In this study we divided 26 subareas where survivors are searched area by area. We have assumed a random time for each SAR operation before a new patient is found. The set time is between 15 to $45 \mathrm{~min}$. In addition, when activities are conducted during night time we doubled the time needed for SAR operations to find the next patient. The sweeping order of subareas can vary. Here we explore 3 cases (see sec. 4).

In addition, the model considers some fix parameters related to the time spend in certain activities. For instance, triage task to one person is set to a random time between 1 or 2 minutes. Also the average time for ambulance dispatch and carry-on of patients into the ambulance has been reported to an average of $2.5 \mathrm{~min}$ and $8.6 \mathrm{~min}$, respectively[11], here we used $3 \mathrm{~min}$ and $9 \mathrm{~min}$.

\section{Simulation cases}

We have simulated three days of disaster response activities including SAR and transportation of patients to the IRCH. The reference event corresponds to the 2011 GEJET and the time of the event was 2:46 pm (JST), then, we assumed a period of one hour and minutes for headquarters to be established and then SAR and transportation activities to get started. Thus, our simulation starts from 4:00 pm until the end of a third day after the event, this is 56 hours of simulation. We tested three cases of SAR patterns to evaluate the rapidity of finding and dispatching survivors from the affected area to the IRCH.

1. Case A.- In this case the order of search starts from the areas closest to the gathering point of SAR teams (i.e. the Firefighters Department) towards the west south of the study area (Fig 3a). Here, the expected advantage would be the fastest exploration of easy-to-reach zones to then increase difficulty due to deeper water and expected larger damage. One may argue the suitability of this sweeping pattern if expectations are that a larger number of serious and severe injury patients would be found towards the coast where deeper tsunami inundation and larger damage occurred. However, on an earthquake and tsunami event, survivors due to collapsed structures in shallow inundated zones or survivors swept inland by tsunami can also be considered, and the pattern in this case would make more sense. Unfortunately very few studies have looked into the spatial distribution of survivors or patients found during post disaster response, possibly due to the difficulties posed in the midst of a disaster to collect such information.

2. Case B.- In this case SAR operations begin near to the hospital location and in shallower areas into 

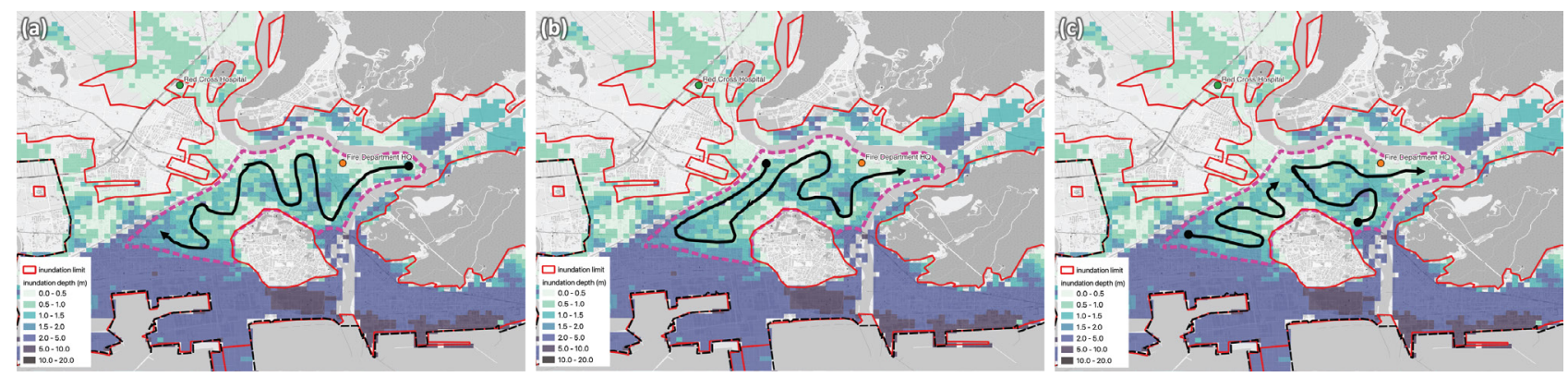

Figure 3: Three search patterns for SAR teams. (a) Case A: easy access prioritization; (b) Case B: closest transportation prioritization; (c) Case $\mathrm{C}$ : deeper inundation prioritization.

deeper inundation zones and zigzagging north-south towards the east (Fig 3b). Here, the main criteria for search is to speed up initial patient transport tasks by prioritizing areas near to the hospital. Similarly, this pattern aims to go as fast as possible to highly affected areas where possibly more injuries are gathered. In addition, alternating between deeper and shallow inundated areas where differences in easiness of mobility may contribute to less fatigue to SAR teams and higher efficiency in the task.

3. Case C.- In this case the SAR operation focuses on deeper inundated zones first, then the action moves inland towards shallower inundated areas (Fig 3c). This means that SAR activities will start at the farthest point from the dispatching point (i.e Firefighters Department) and the hospital where patients are transported.

\section{Results and Discussion}

Table 3 shows the average of patients' searched and rescued from the tsunami affected area. Slight differences among cases are shown. For instance, Case A appears to be the pattern giving better results with a higher average value. Apparently the three cases of SAR operations does not present significant differences on the timing of patients' transportation.

Table 3: Number of patients with severe or medium level injuries transported within 3 days in each case of SAR pattern.

\begin{tabular}{|l|c|c|c|}
\cline { 2 - 4 } \multicolumn{1}{c|}{} & Case A & Case B & Case C \\
\hline Average & 210 & 206 & 204 \\
\hline Maximum & 223 & 223 & 223 \\
\hline Minimum & 191 & 184 & 184 \\
\hline Std. Dev. & 5.19 & 4.38 & 4.94 \\
\hline
\end{tabular}

Table 4 shows the number of patients transported each day on the three cases in study, as well as the IRCH records of the event. The model shows underestimation on the first days of activity and overestimation in the last day. Overall day by day discrepancies are balanced out in the total
Table 4: Number of patients transported to the IRCH according to triage level and the days after the event. Three cases were compared to the IRCH record of patients and their medical level of need.

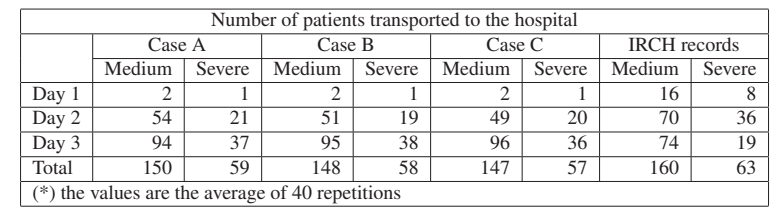

sum where the model shows good agreement. Some of the reasons for such differences can be explained by the limitations of the model to represent other activities that contribute to the transportation of patients. For instance, the JSDF transported patients via helicopter and several patients where transported by private vehicles without the assistance of emergency responders.

In addition, outcomes of Cases A, B and C differ little due to the reduced size of the area covered in SAR and transportation. Results at larger areas might be different for the three cases since patients' location would be more scattered and distances and time for finding and move would be longer. The overall outcome for patients transported within 56 hours are similar to the IRCH records. This is expected due to the nature of the input in the model, however, reaching such ceiling within 3 days simulation verifies the model scheduling activities (agent behavior) within a reasonable marginal error.

It is possible that in the real situation, when limited resources where available at the beginning self-response and other means of transportation contributed to higher number of patients being transported compared to the model. Similarly, on the third day, when resources are plenty, the model compensates for the number of patients, while in the real event, by that time plenty of resources would have been more than enough to move the remaining survivors.

\section{Conclusions}

This study has developed an agent-based model to simulate search and rescue operations together with transportation of patients to the hospital. A portion of Ishinomaki city was taken as a case study to compared the outcomes 
of the model to the actual data of patients that were received at the Ishinomaki Red Cross Hospital.

We averaged the outcome values of 40 simulation runs showing agreement to the actual records. At a finer level, the model presents underestimated results during the first two days of relief activities, while overestimation is found on the third day. Limitations on the model, as well as a possible number of self-response via private means of transportation might explain such discrepancies. Further improvement in the model to incorporate other means of transportation is necessary, however data for verification might be scarce in this case.

\section{References}

[1] World Health Organization. The Great East Japan Earthquake. A story of a devastating natural disaster, a tale of human compassion. 2012

[2] Ranghieri, Federica and Ishiwatari, Mikio. Learning from megadisasters: lessons learnt from the Great East Japan earthquake and tsunami. Economics and Policy of Energy and the Environment, 3(3), 5-17, 2015.

[3] Egawa, Shinichi and Suda, Tomomi and JonesKonneh, Tracey Elizabeth Claire and Murakami, Aya and Sasaki, Hiroyuki. Nation-Wide Implementation of Disaster Medical Coordinators in Japan. The Tohoku Journal of Experimental Medicine, 243(1), 1-9, 2017.

[4] Ishii, Tadashi. Medical response to the Great East Japan Earthquake in Ishinomaki City. Western Pacific Surveillance and Response Journal, 2(4), 2011.
[5] Serikawa, Tomoki and Seto, Shuji and Suppasri, Anawat and Imamura, Fumihiko. Spatial Distribution of Causes of Death in the 2011 Tohoku Tsunami at Ishinomaki City, Miyagi Prefecture. Journal of Disaster Research, 15(7), 943-958, 2020.

[6] Koresawa, Atsushi. Evidence-Based Analysis of Search and Rescue Operations Following the Great East Japan Earthquake. Journal of Disaster Research, 8, 746-755, 2013.

[7] Araki, Yasuhiro. A study for modeling transportation activities of casualties by Multi Agent Simulation in consideration of the Niigataken Chuetsu-oki Earthquake in 2007. Journal of Social Safety Science, 10(11), 1-9, 2008. (in Japanese).

[8] Ishinomaki District Wide Area Administrative Office Fire Department. Great East Japan Earthquake 3.11 Ishinomaki Wide Area Firefighting Activity Record. 2012. (in Japanese).

[9] Japanese Red Cross Society Planning and Public Relations Office. Great East Japan Earthquake-All records from relief activities to reconstruction assistance. 2013. (in Japanese).

[10] Ishinomaki City. Great East Japan Earthquake: History of Ishinomaki City. 2017 (in Japanese).

[11] Kataoka, Motomune and Yoshii, Toshio and Futagami, Toru and Oguchi, Takashi. Development of a model of the transportation time sent to a hospital by ambulance. Seisan Kenkyu 67(2), 137-142, 2015. (in Japanese). 